\title{
BMJ Open Clinical outcomes at 12 months and risk of inflammatory bowel disease in patients with an intermediate raised fecal calprotectin: a 'real-world' view
}

\author{
Michael McFarlane, ${ }^{1}$ Samantha Chambers, ${ }^{1}$ Ahmad Malik, ${ }^{1}$ Bee Lee, ${ }^{2}$ \\ Edmond Sung, ${ }^{3}$ Chuka Nwokolo, ${ }^{1}$ Norman Waugh, ${ }^{4}$ Ramesh Arasaradnam ${ }^{1,5}$
}

To cite: McFarlane $M$, Chambers S, Malik A, et al. Clinical outcomes at 12 months and risk of inflammatory bowel disease in patients with an intermediate raised fecal calprotectin: a 'real-world' view. BMJ Open 2016;6: e011041. doi:10.1136/ bmjopen-2016-011041

- Prepublication history and additional material is available. To view please visit the journal (http://dx.doi.org/ 10.1136/bmjopen-2016011041).

Received 7 January 2016 Revised 23 February 2016 Accepted 9 March 2016

\section{CrossMark}

${ }^{1}$ Department of Gastroenterology, University Hospital Coventry \& Warwickshire, Coventry, UK ${ }^{2}$ Department of Gastroenterology, Warwick Hospital, Warwick, UK ${ }^{3}$ Department of Gastroenterology, George Eliot Hospital, Nuneaton, UK ${ }^{4}$ Department of Warwick Evidence, University of Warwick, Coventry, UK ${ }^{5}$ Clinical Sciences Research Institute, University of Warwick, Coventry, UK

Correspondence to Professor Ramesh Arasaradnam; r.arasaradnam@warwick.ac.uk

\section{ABSTRACT}

Objectives: A recent systematic review confirmed the usefulness of fecal calprotectin (FC) in distinguishing organic (inflammatory bowel disease (IBD)) from non-organic gastrointestinal disease (irritable bowel syndrome (IBS)). FC levels $<50 \mu \mathrm{g} / \mathrm{g}$ have a negative predictive value $>92 \%$ to exclude organic gastrointestinal (GI) disease. Levels $>250 \mu \mathrm{g} / \mathrm{g}$ correlate with endoscopic IBD disease activity; sensitivity $90 \%$. We aimed to determine clinical outcomes in intermediate raised FC results $(50-250 \mu \mathrm{g} / \mathrm{g})$.

Setting: Primary care general practices in Coventry and Warwickshire, and 3 secondary care hospitals.

Participants: 443 FC results in adults ( $>16$ years old) were reviewed from July 2012 to October 2013. Clinical data was collected from hospital databases and general practitioners. Long-term clinical data was available in 41 patients (out of 48).

Primary and secondary outcome measures: The number of new diagnoses of IBD, IBS and other diagnoses for the intermediate group. The number referred and discharged from secondary care.

Results: A new IBD diagnosis was made in 19\% $(n=8)$ of intermediate results ( $1 \%$ of normal and $38 \%$ of raised results). $5 \%(n=2)$ of intermediate results had known IBD in remission. A new IBS diagnosis was made in $27 \%(n=11)$ of intermediate results, while $34 \%$ $(n=14)$ remained undiagnosed, although 8 of these were not referred to secondary care.

Conclusions: FC testing remains useful in aiding diagnosis of organic GI conditions. However, unlike negative and strongly positive FC results, intermediate FC results lead to a mixture of diagnoses. The OR of a new diagnosis of IBD for an intermediate result compared to normal FC result was 26.6, while an intermediate $\mathrm{FC}$ result gave an OR of 0.54 for a new IBS diagnosis compared to normal FC. For intermediate FC results, 1 in 3 patients remained in secondary care after 12 months with an OR of 3.6 compared to a normal FC result.

\section{INTRODUCTION}

Calprotectin is a calcium binding protein of the S100 family, found mainly in neutrophils
Strengths and limitations of this study

- Twelve-month clinical follow-up data of intermediate fecal calprotectin results.

- A 'real-world' view of the usefulness of FC testing in primary and secondary care-it is not being used in an entirely appropriate manner.

- A 'real-world' view of clinical outcomes-we don't always find the answer.

- Heterogeneous data sources means data is not as complete as it could be, for example, medication information incomplete.

but also in other white blood cells. ${ }^{1}$ Inflammation results in neutrophil activation and a subsequent release of calprotectin protein. $^{23}$ The use of fecal calprotectin (FC) has particular interest as a non-invasive biomarker in the initial screening and monitoring of patients with suspected or known inflammatory bowel disease (IBD). ${ }^{4}$ It is of particular use in the distinction between inflammatory gastrointestinal (GI) conditions such as IBD, from non-organic conditions such as irritable bowel syndrome (IBS).$^{5} \mathrm{FC}$ testing also has a growing role in the monitoring of IBD activity in response to treatment, although long-term data on the clinical advantages of this approach are not yet available. ${ }^{6}$

Current National Institute of Health and Care Excellence (NICE) and manufacturer guidelines for the cut-off levels of FC in assays are that levels of $<50 \mu \mathrm{g} / \mathrm{g}$ of feces suggest that there is no active inflammation present within the GI mucosa. ${ }^{5} 7$ NICE reported that for most of the studies they reviewed, sensitivity and specificity were over $80 \%$, where a cut-off of $50 \mu \mathrm{g} / \mathrm{g}$ was used, and most positive and negative predictive values were $70-90 \% .^{7}$ One recent study found that a cut-off of $50 \mu \mathrm{g} / \mathrm{g}$ gives a sensitivity and specificity of $88 \%$ and $78 \%$, 
respectively, with a negative predictive value of $>92 \%$ to exclude organic GI disease. ${ }^{8}$ A cut-off value of $100 \mu \mathrm{g} / \mathrm{g}$ has previously been suggested, and while this increases sensitivity to $97 \%$, specificity falls to $76 \%$, with a negative predictive value of $97 \%$, and a positive predictive value of $75 \% .{ }^{8}$ A cohort study involving consecutively referred new patients with chronic diarrhoea proposed that a cut-off of $8 \mu \mathrm{g} / \mathrm{g}$ provides near $99 \%$ sensitivity in detecting organic disease but at the cost of poor specificity. ${ }^{9}$ In this study, no patients were diagnosed with IBD with FC levels of $50 \mu \mathrm{g} / \mathrm{g}$ or less, although this was a small study, hence, only a few patients overall with IBD. Another study found that no patients with an FC result of $<100 \mu \mathrm{g} / \mathrm{g}$ had IBD. ${ }^{10}$ A systematic review of the use of FC, which informed the NICE diagnostic assessment group, found that most of the available evidence for FC use in IBD is based on a cut-off value of $50 \mu \mathrm{g} / \mathrm{g}$ which reduces the number of false negatives while maintaining cost-effectiveness. ${ }^{5} 7$

An FC value of $>250 \mu \mathrm{g} / \mathrm{g}$ has been demonstrated to correspond with endoscopically and histologically active IBD. This cut-off level provides a sensitivity of $90 \%$ and specificity of $76 \%$ for excluding IBD, and also determining if known disease was quiescent. ${ }^{8} \mathrm{~A}$ recent meta-analysis into the utility of FC testing for monitoring disease activity showed that for known IBD, a cut-off level of $50 \mu \mathrm{g} / \mathrm{g}$ gave a pooled sensitivity and specificity of $92 \%$ and $60 \%$ for determining if disease was quiescent, a cut-off of $100 \mu \mathrm{g} / \mathrm{g}$ gave $84 \%$ and $66 \%$, while a cut-off of $250 \mu \mathrm{g} / \mathrm{g}$ gave $80 \%$ and $82 \%$, respectively. ${ }^{11}$ See online supplementary appendix 1 for a tabulated form of the studies involving FC cut-offs for screening for IBD.

Despite the increasing number of studies involving utility of FC there has been limited study on patients who have a FC value which falls within the 'intermediate' group defined as levels of $50-250 \mu \mathrm{g} / \mathrm{g}$ of feces. While these patients may still have underlying organic GI disease, current opinion suggests that the FC assay should be repeated 4-6 weeks later to determine if inflammation is improving or worsening. Although in reality an intermediate result may still be referred to secondary care if clinical suspicion is high for underlying IBD, our local guidance is in accordance with these ranges (see figure 1).

The aim of this study was to determine the 12-month clinical outcomes of patients with an intermediate raised FC value. There have been, to the best of our knowledge, no published studies which look at clinical outcomes in this group of patients and proportions remaining in secondary/tertiary care follow-up.

\section{Recommended Primary Care Guidelines}

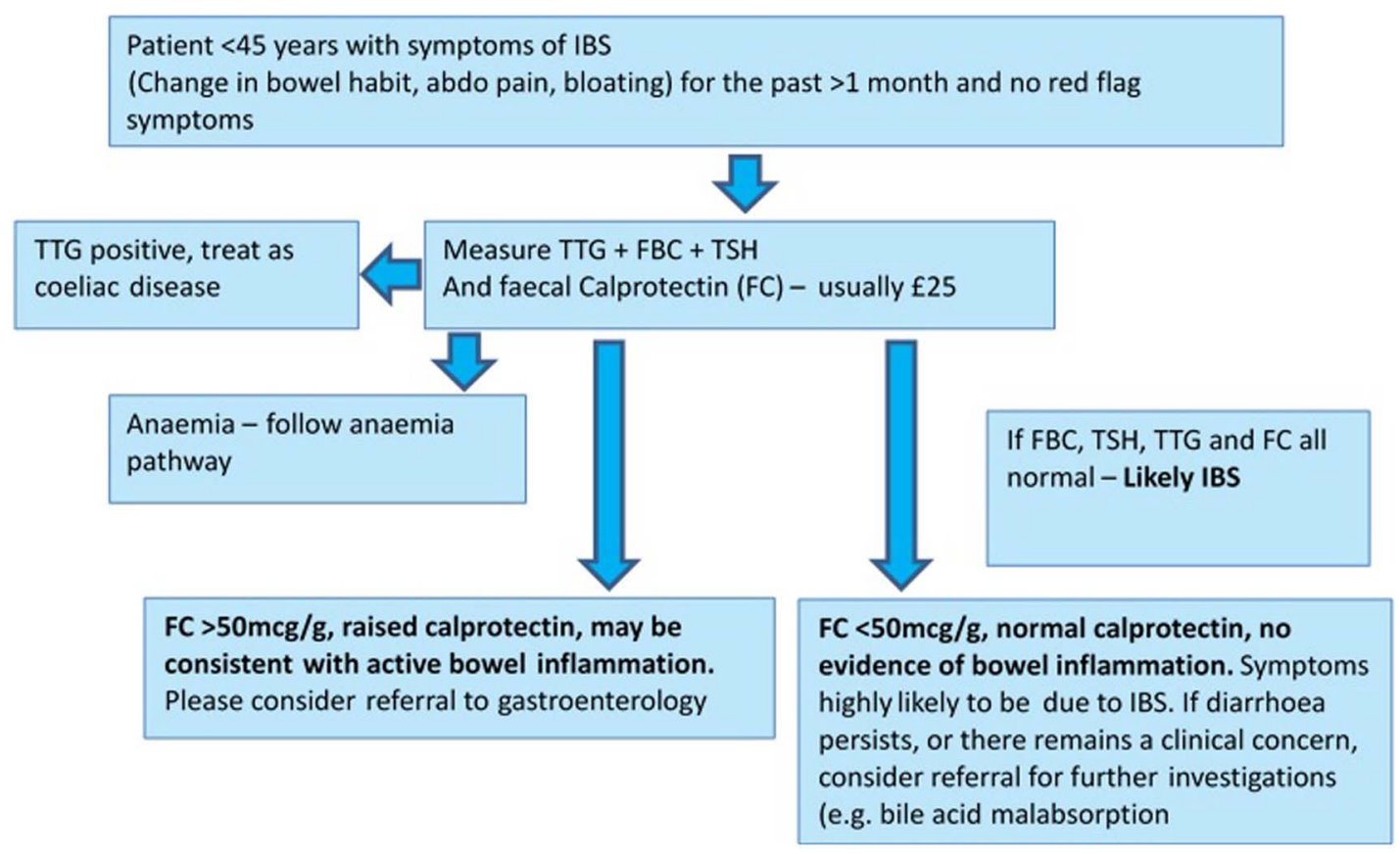

Figure 1 Local Guidelines for GPs to determine the usefulness of FC in those with chronic diarrhoea.

- Do NOT use FC for bowel cancer.

- Do NOT use FC for infection.

- DO give your patient a labelled stool pot; 2p-sized amount of sample is sufficient (solid stool is best).

- DO ask them to bring it to their local hospital/GP to send to BIOCHEMISTRY (not microbiology)—stable at room temp.

- DO fill in as much clinical information as possible, including symptoms and duration.

- DO send a separate sample to microbiology for M, C and S unless infection has already been excluded. 


\section{MATERIALS AND METHODS}

\section{FC samples}

FC test results from July 2012 to October 2013 in the University Hospital Coventry \& Warwickshire (UHCW) pathology database were reviewed. Tests were requested by primary care and secondary/tertiary care physicians from the Warwickshire region, a catchment population of 1 million. Outcomes at 12 months were linked to index FC result. We have not excluded patients with other GI conditions, organic or inorganic, as we want to provide an insight into how FC testing is currently being used in real-world practice.

\section{Clinical data collection}

Clinical information was collected from review of the central base UHCW (tertiary catchment of $1 \mathrm{~m}$ population) clinical results reporting system and the corresponding systems in neighbouring district hospitals (Warwick and George Eliot Hospitals). If no information was found then general practitioners (GPs) were contacted for further details.

Owing to the heterogeneous nature of the data collection sources, clinical details such as symptoms and medication which may affect FC values (eg, non-steroidal anti-inflammatory drugs (NSAIDs) or proton pump inhibitors (PPIs)) were often unavailable or poorly recorded, especially for the general practice patients. These factors were not considered here due to the lack, or incompleteness, of the data.

\section{FC sample analysis}

Standardised laboratory protocol at UHCW for FC analysis was used. A $100 \mathrm{mg}$ quantity of stool was weighed and dispensed into an analysis pot using a $10 \mu \mathrm{L}$ inoculation loop. As per local protocol, first morning sample was requested. The exact weight was recorded and $5 \mathrm{~mL}$ of extraction buffer added. The samples were then vortexed for $30 \mathrm{~min}$ in order to ensure complete dissolution, and then centrifuged using an Eppendorf centrifuge. The supernatant was then removed for analysis using the Immunodiagnostik PhilCal ELISA method.

\section{Ethics}

Ethical guidance was obtained from the Coventry and Warwick NRES Committee. As this study was evaluating a test already in clinical practice, no further ethical approval was required.

\section{RESULTS}

A total of 495 patients were identified; 52 paediatric patients (aged $<16$ years) were excluded. Of the remaining, there were $365(82 \%)$ with a normal FC result $(<50 \mu \mathrm{g} / \mathrm{g}), 48(11 \%)$ with an intermediate result $(50-250 \mu \mathrm{g} / \mathrm{g})$, and $30(7 \%)$ with a high result $(>250 \mu \mathrm{g} / \mathrm{g})$. Long-term clinical data was available in 41 of the intermediate results. The mean age was 44 years (SD 19.0). There were 17 men (41\%) and 24 women $(59 \%)$. The mean FC for the intermediate cohort was $130 \mu \mathrm{g} / \mathrm{g}$ (SD 58).

Two persons $(4.8 \%)$ were known IBD patients under ongoing secondary care for their condition. They were included as we wished to provide a 'snapshot' of the usage of FC testing in our centre, including that of already known IBD patients under surveillance. A total of $28(68 \%)$ patients with an intermediate $\mathrm{FC}$ result were referred to secondary/tertiary gastroenterology care by their primary care physicians, with 11 (27\%) managed in primary care. Only one patient had a repeat FC sample sent, and this remained moderately raised. The patient subsequently had normal investigations and was discharged.

A total of $29(71 \%)$ patients with an intermediate FC result went on to have a colonoscopy, with $8(20 \%)$ also undergoing a CT scan, and a further 7 (17\%) undergoing an MRI. Only eight patients out of the 41 for whom clinical information was available did not undergo any investigations. These patients were those who were not referred to secondary care. This would suggest that an intermediate result does not reduce the number of colonoscopies or imaging investigations being done.

The diagnoses of intermediate FC results were:

- Eight new cases of IBD diagnosed within the 41 patients (19\%; SE6.1\%) with FC levels ranging from 81 to $218 \mu \mathrm{g} / \mathrm{g}$. All underwent colonoscopies:

- Three $(7.3 \%)$ were diagnosed with Crohn's disease (Montreal Classification: 1 L2 (colonic); 2 L3 (ileocolonic);

- Three $(7.3 \%)$ were diagnosed with ulcerative colitis (UC) (Montreal Classification: 2 E1 (proctitis); 1 E2 (left sided);

- Two $(4.7 \%)$ with IBD unspecified.

- Six $(15 \%)$ were diagnosed with other organic GI conditions, including bile salt malabsorption, diverticular disease and carcinoid.

- Eleven (27\%; SE 7\%) were diagnosed with IBS.

- Fourteen $(34 \%)$ remained undiagnosed, however, 8 $(19 \%)$ of these 14 were not referred to secondary care by their GP. The reasons for this are unknown; their symptoms may have settled spontaneously or the patients themselves may have declined referral:

- Of the six undiagnosed patients (15\%) who were referred to secondary care

- Three $(7.3 \%)$ were discharged after investigation with no cause found for the raised FC, with their symptoms having resolved;

- Three (7.3\%) were still under investigation for other causes of the intermediate FC result, for example, small bowel bacterial overgrowth.

See table 1 and figure 2 for a further breakdown of these results.

The OR for a new diagnosis of IBD with an intermediate result compared to a normal FC result was 26.6. The OR of a new diagnosis of IBS with an intermediate result compared to a normal FC result was 0.54 . The OR of remaining in secondary care 12 months after diagnosis 
Table 1 Patient demographics and FC values per FC category of low, intermediate and high over 12 months

\begin{tabular}{llll}
\hline & FC $<\mathbf{5 0} \boldsymbol{\mu \mathbf { g }} \mathbf{g}$ & $\mathbf{F C} \mathbf{5 0 - 2 5 0} \boldsymbol{\mu \mathbf { g } / \mathbf { g }}$ & $\mathbf{F C}>\mathbf{2 5 0} \boldsymbol{\mu \mathbf { g } / \mathbf { g }}$ \\
\hline Mean age (SD) & $39(15)$ & & \\
& $38(13)$ & $44(19)$ & $39(16)$ \\
Male:female (\%) & $36: 64$ & & \\
& $35: 65$ & $42: 58$ & $35: 65$ \\
Mean FC $\mu \mathrm{g} / \mathbf{g}(\mathrm{SD})$ & $22(5.0)$ & $130(58)$ & $784(573)$ \\
Referred to secondary care (n) & $66.3 \%(138)$ & $68.3 \%(28)$ & $57.7 \%$ \\
Remain in secondary care at 12 months postindex FC (n) & $9.1 \%(19)$ & $34.1 \%(14)$ & $73.1 \%(15)$ \\
Diagnoses & & & \\
Pre-existing IBD (n) & $3.4 \%(7)$ & $4.8 \%(2)$ & $23 \%(6)$ \\
New diagnosis IBD (n) & $1.0 \%(2)$ & $19 \%(8)$ & $38.4 \%(10)$ \\
Other organic GI diagnosis (n) & $13 \%(27)$ & $14.6 \%(6)$ & $3.8 \%(1)$ \\
Diagnosis IBS (n) & $40.4 \%(84)$ & $26.8 \%(11)$ & $7.7 \%(2)$ \\
Undiagnosed or awaiting diagnosis (n) & $41.8 \%(87)$ & $34.1 \%(14)$ & $26.9 \%(7)$ \\
\hline FC, fecal calprotectin; GI, gastrointestinal; IBD, inflammatory bowel disease; IBS, irritable bowel syndrome. &
\end{tabular}

with an intermediate FC result was 3.6 compared to a normal FC result.

Twelve months after their initial FC test, $14(34 \%)$ of the cohort remained under ongoing secondary/tertiary care for their conditions, with 14 (34\%) discharged back to their primary care physicians. The remainder were managed in primary care.

Subgroup analysis of the intermediate patients was conducted below and above $100 \mu \mathrm{g} / \mathrm{g}$ threshold; 18 $(44 \%)$ of the intermediate group had $\mathrm{FC}<100 \mu \mathrm{g} / \mathrm{g}$, while $23(56 \%)$ had FC $>100 \mu \mathrm{g} / \mathrm{g}$. For the $<100 \mu \mathrm{g} / \mathrm{g}$ group, $14(78 \%)$ were in primary care 12 months after index FC test compared with $13(57 \%)$ of $>100 \mu \mathrm{g} / \mathrm{g}$ group at the same time point. Three $(17 \%)$ with $<100 \mu \mathrm{g} / \mathrm{g}$ received a new diagnosis of IBD compared with five $(22 \%)$ with $>100 \mu \mathrm{g} / \mathrm{g}$ group. One patient in each of the $<100 \mu \mathrm{g} / \mathrm{g}$ group and the $>100 \mu \mathrm{g} / \mathrm{g}$, respectively, were known IBD patients, with $2(11 \%)$ and 3 $(13 \%)$ patients, respectively, being diagnosed with non-IBD organic GI conditions including bile acid malabsorption, diverticular disease and rectal carcinoid; $28 \%$ of $<100 \mu \mathrm{g} / \mathrm{g}$ cohort and $26 \%$ of $>100 \mu \mathrm{g} / \mathrm{g}$ cohort were diagnosed with IBS; while $38 \%$ of $<100 \mu \mathrm{g} / \mathrm{g}$ and $35 \%$ of $>100 \mu \mathrm{g} / \mathrm{g}$ groups did not receive a formal diagnosis. No

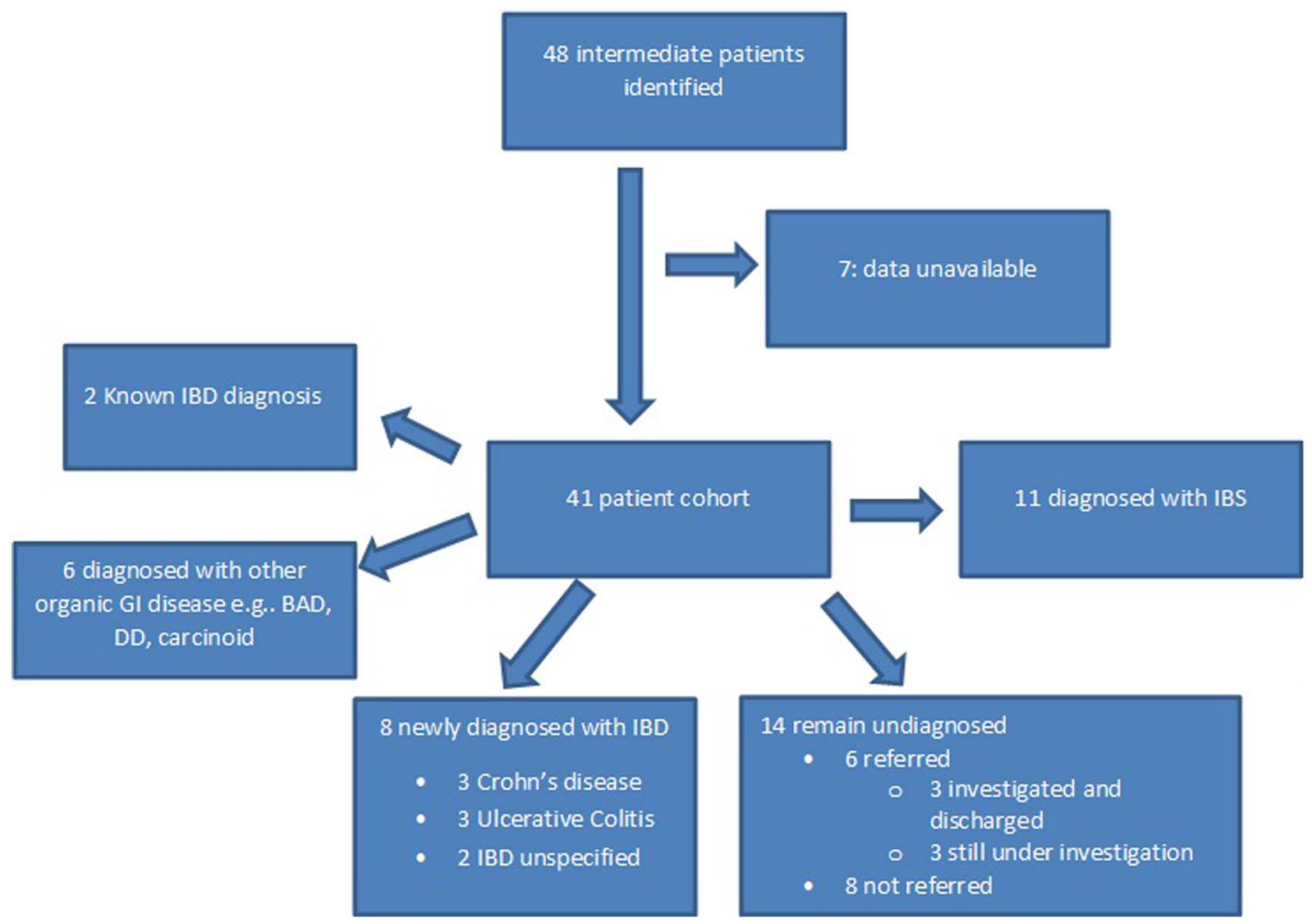

Figure 2 Flow chart of Intermediate Fecal Calprotectin (FC) diagnoses. 
Table 2 Outcome subanalysis of FC intermediate groups; $<100$ and $>100 \mu \mathrm{g} / \mathrm{g}$

\begin{tabular}{lll}
\hline & $\begin{array}{l}\mathbf{F C}<100 \\
(\mathbf{n}=18)\end{array}$ & $\begin{array}{l}\text { FC>100 } \\
(\mathbf{n}=\mathbf{2 3})\end{array}$ \\
\hline $\begin{array}{l}\text { In primary care 12 months } \\
\text { postindex FC (\%) }\end{array}$ & $78 \%(n=14)$ & $57 \%(n=13)$ \\
New diagnosis of IBD & $17 \%(n=3)$ & $22 \%(n=5)$ \\
Known IBD & $5.6 \%(n=1)$ & $4.3 \%(n=1)$ \\
Other organic GI diagnosis & $11 \%(n=2)$ & $13 \%(n=3)$ \\
IBS diagnosis & $28 \%(n=5)$ & $26 \%(n=6)$ \\
others & $38.4 \%$ & $34.7 \%$ \\
\hline
\end{tabular}

FC, fecal calprotectin; GI, gastrointestinal; IBD, inflammatory bowel disease; IBS, irritable bowel syndrome.

statistical differences were noted between these two groups. See table 2 for the subgroup analysis results.

\section{DISCUSSION}

Our study provides some insight into the 'real-world' use of FC testing in primary and secondary care. An intermediate or raised FC result increases the probability of remaining under secondary care 12 months after the index FC test-34\% of intermediate results were still in secondary care 12 months after index FC, compared with $9.1 \%$ of the normal group and $73 \%$ for the raised group. This reflects the increasing likelihood of a diagnosis of IBD or another organic GI disease with intermediate or raised $\mathrm{FC}$ results.

A new diagnosis of IBD was made in $19 \%$ of patients with an intermediate $\mathrm{FC}$ result, compared with $1 \%$ for normal results and $37 \%$ for raised results. These findings suggest that an initial intermediate $\mathrm{FC}$ result gives an OR of 26.6 for receiving a diagnosis of IBD compared to a normal FC result, and an OR of 3.6 for remaining in secondary care 12 months after index FC result for intermediate results compared to a normal FC result. This would seem to increase the evidence for suggesting that intermediate $\mathrm{FC}$ results should undergo retesting 4-6 weeks later before referral to secondary care is made. Of note, a third in this group had no final diagnosis at 12 months, with most of these arising from primary care. Unfortunately, no repeat FG specimens were sent by primary care to determine if the intermediate result was consistent or transient.

Subgroup analysis of the intermediate group does not show a clinically significant difference between the $<100 \mu \mathrm{g} / \mathrm{g}$ group and the $>100 \mu \mathrm{g} / \mathrm{g}$. There were similar numbers for: new IBD diagnoses made $(17 \%$ $(<100 \mu \mathrm{g} / \mathrm{g})$ vs $22 \%(>100 \mu \mathrm{g} / \mathrm{g}))$; other organic GI diseases $(11 \%$ vs $13 \%)$, and IBS diagnoses (28\% vs $26 \%$ ). Therefore, based on this study group and usefulness of the ELISA Phical methods, there is no suggestion that the cut-off value for a normal FG result alters diagnostic yield if it were raised to $100 \mu \mathrm{g} / \mathrm{g}$.

NICE guidelines on FC usage do not currently set an age cut-off for FC testing. NICE IBS guidelines use an age cut-off $>50$ as a red flag for change in bowel habit. Local guidelines state that FC testing should not be used over the age of 40 years due to the rising incidence of bowel cancer. Rather, such patients with a change in bowel habit or diarrhoea should undergo endoscopic investigation. In this study, we found that the mean age of patients undergoing FC testing was 39 years, with $40 \%$ older than 40 years and $19 \%$ older than 50 years. Within the intermediate group, $46 \%$ met local guidelines on FC testing and $66 \%$ met NICE guidelines' recommended red flag cut-off of $<50$ years. The oldest patient to undergo FC testing was 89 years. This would suggest that there needs to be greater education and awareness about the strengths and limitations of FC testing in aiding with diagnosis or exclusion of organic GI diseases, principally IBD.

A limitation in this study is the heterogeneous clinical data, which comprised both primary and secondary care data, which resulted in incomplete clinical details such as symptoms and medications, including NSAIDs, across all FC ranges. However, the objectives of this study were to understand the way in which FC testing is currently being used in our region. We have attempted to provide a 'real-world' snapshot of how FC testing is being used in primary and secondary care settings, with some not receiving a definitive diagnosis. NICE appraisal did raise the issue of spectrum bias because most of the studies in the assessment report ${ }^{5}$ came from secondary care settings. Unfortunately, the numbers of patients in our study, and the lack of data on long-term outcomes on a substantial number of those with normal results, make it impossible for us to analyse screening parameters, such as NPV, separately by source.

Further studies are needed to determine the longterm clinical outcomes in patients undergoing FC testing in both primary and secondary care, to aid interpretation of intermediate raised FG results. It could encourage the use of repeat FC testing for intermediate results, rather than immediate referral to secondary care for further investigation. Better education of the indications, potential confounding drugs, and age issues with FC testing would provide more efficient use of FC testing and would allow for far clearer interpretation of the true meaning of intermediate results.

Acknowledgements The authors thank the UHCW pathology department and the GPs of Coventry and Warwickshire for their cooperation in this study.

Contributors MM coordinated the project, collected and analysed the data and wrote the manuscript. SC collected and analysed the data, wrote the manuscript. AM, BL, ES collected the data $\mathrm{CN}$ oversaw the project, contributed to the paper content. NW analysed the data, co-wrote the manuscript. RA coordinated the project, cowrote the manuscript.

Funding This research received no specific grant from any funding agency in the public, commercial or not-for-profit sectors.

Competing interests None declared.

Provenance and peer review Not commissioned; externally peer reviewed.

Data sharing statement No additional data are available. 
Open Access This is an Open Access article distributed in accordance with the Creative Commons Attribution Non Commercial (CC BY-NC 4.0) license, which permits others to distribute, remix, adapt, build upon this work noncommercially, and license their derivative works on different terms, provided the original work is properly cited and the use is non-commercial. See: http:// creativecommons.org/licenses/by-nc/4.0/

\section{REFERENCES}

1. Røseth AG, Fagerhol MK, Aadland E, et al. Assessment of the neutrophil dominating protein calprotectin in feces. A methodologic study. Scand J Gastroenterol 1992;27:793-8.

2. Aomatsu T, Yoden A, Matsumoto K, et al. Fecal calprotectin is a useful marker for disease activity in pediatric patients with inflammatory bowel disease. Dig Dis Sci 2011;56:2372-7.

3. Stríz I, Trebichavský I. Calprotectin-a pleiotropic molecule in acute and chronic inflammation. Physiol Res 2004;53:245-53.

4. van Rheenen PF, Van de Vijver E, Fidler V. Faecal calprotectin for screening of patients with suspected inflammatory bowel disease: diagnostic meta-analysis. BMJ 2010;341:c3369.

5. Waugh N, Royle P, Cummins E, et al. Faecal calprotectin testing for differentiating amongst inflammatory and non-inflammatory bowel diseases: systematic review and economic evaluation. Health Technol Assess 2013;17:xv-xix, 1-211.

6. Sipponen T, Kärkkäinen P, Savilahti E, et al. Correlation of faecal calprotectin and lactoferrin with an endoscopic score for Crohn's disease and histological findings. Aliment Pharmacol Ther 2008;28:1221-9.

7. National Institute for Health and Clinical Excellence. Faecal Calprotectin diagnostic tests for inflammatory diseases of the bowel. NICE diagnostic guidance (DG11). 2013.

8. Dhaliwal A, Zeino Z, Tomkins C, et al. Utility of faecal calprotectin in inflammatory bowel disease (IBD): what cut-offs should we apply? Frontline Gastroenterol 2015;6:14-19.

9. Banerjee A, Srinivas M, Eyre R, et al. Faecal calprotectin for differentiating between irritable bowel syndrome and inflammatory bowe disease: a useful screen in daily gastroenterology practice Frontline Gastroenterol 2013; in press.

10. Koulaouzidis A, Douglas S, Rogers MA, et al. Fecal calprotectin: a selection tool for small bowel capsule endoscopy in suspected IBD with prior negative bi-directional endoscopy. Scand J Gastroenterol 2011;46:561-6.

11. Lin JF, Chen JM, Zuo JH, et al. Meta-analysis: fecal calprotectin for assessment of inflammatory bowel disease activity. Inflamm Bowel Dis 2014:20:1407-15. 